PROCEEDINGS OF THE

AMERICAN MATHEMATICAL SOCIETY

Volume 125, Number 6, June 1997, Pages 1659-1665

S 0002-9939(97)03545-4

\title{
SOME HARMONIC FUNCTIONS ON MINKOWSKI SPACE
}

\author{
P. F. GLEZEN AND R. C. PENNER
}

(Communicated by Peter Li)

\begin{abstract}
This note presents elementary geometric descriptions of several simple families of harmonic functions on the upper sheet of the unit hyperboloid in Minkowski three-space. As is briefly discussed here, these calculations grew out of an earlier attempt to construct Poincaré series on punctured surfaces using Minkowski geometry.
\end{abstract}

\section{INTRODUCTION}

The material in this note grew out of an attempt (discussed briefly in $\S 3$ below) some time ago to construct harmonic functions on a punctured surface in terms of Minkowski geometry. Indeed (in [EP] and [P1]), we associate a convex body in Minkowski three-space to a punctured Riemann surface, and Minkowski lengths of extreme edges of this body were seen (in $[\mathrm{P} 1]$ ) to give a coordinatization of the (decorated) Teichmüller space. As we show in Proposition 2 of this note, these coordinates admit a natural extension to harmonic coordinates on the upper sheet $\mathbb{H}^{+}$of the unit hyperboloid in Minkowski space; furthermore, the harmonic conjugate is computable in these coordinates, and the associated analytic function on $\mathbb{H}^{+}$is the one naturally associated to a suitable pair of points in the light-cone as we shall see.

There also arose another family of harmonic functions generalizing the usual Green's function on $\mathbb{H}^{+}$, where this family is parametrized by suitable pairs of points in Minkowski space. These harmonic functions are described in Proposition 1, the proof of which depends upon a family of solutions to the partial differential equation on conformal factors in the solution to the Yamabe problem; this last family is parametrized by points of Minkowski three-space itself. Finally, in Proposition 3, we describe a picture (which is certainly standard but anyway deserves to be better known) of Poisson kernel for $\mathbb{H}^{+}$in terms of the geometry of Minkowski space.

It is not that we are constructing "new" families of harmonic functions here, but rather that "known" harmonic functions admit essentially linear expressions in our coordinates on Minkowski space.

The techniques of this paper are entirely elementary and largely computational, and there evolves a (presumably well-known) interplay between hyperbolic geometry and harmonic analysis. The second-named author thanks Itzhak Bars and Paul Yang for helpful discussions.

Received by the editors April 7, 1995.

1991 Mathematics Subject Classification. Primary 30Cxx, 30Fxx.

The second author was partially supported by the National Science Foundation.

(C) 1997 American Mathematical Society 


\section{Notation}

The reader desiring further information on (some of) the material in this section is referred to $\S 2$ of $[\mathrm{P} 1]$.

Let $\mathbb{M}$ denote Minkowski three-space, so $\mathbb{M}$ is identified with $\mathbb{R}^{3}$ (with its standard coordinates $x, y$, and $z$ ) and is endowed with the pairing

$$
\left\langle\left(x_{1}, y_{1}, z_{1}\right),\left(x_{2}, y_{2}, z_{2}\right)\right\rangle=x_{1} x_{2}+y_{1} y_{2}-z_{1} z_{2} .
$$

The "height" of a point $\vec{p}=(x, y, z) \in \mathbb{M}$ is defined to be simply $h(\vec{p})=z$. Let us also define the following distinguished subsets of $\mathbb{M}$ :

$$
\begin{aligned}
L & =\{\vec{p} \in \mathbb{M}:\langle\vec{p}, \vec{p}\rangle=0\}, \\
L^{+} & =\{\vec{p} \in L: h(\vec{p})>0\}, \\
\mathbb{H} & =\{\vec{p} \in \mathbb{M}:\langle\vec{p}, \vec{p}\rangle=-1\}, \\
\mathbb{H}^{+} & =\{\vec{p} \in \mathbb{H}: h(\vec{p})>0\},
\end{aligned}
$$

and

$$
H=\{\vec{p} \in \mathbb{M}:\langle\vec{p}, \vec{p}\rangle=1\} .
$$

Of course, $\mathbb{H}^{+}$inherits a Riemannian metric from $\langle\cdot, \cdot\rangle$ and gives a model for the hyperbolic plane; furthermore, the assignments

$$
\begin{aligned}
L^{+} & \rightarrow\left\{\text { horocycles in } \mathbb{H}^{+}\right\}, \\
\vec{u} & \mapsto\left\{\vec{w} \in \mathbb{H}^{+}:\langle\vec{w}, \vec{u}\rangle=-1\right\},
\end{aligned}
$$

and

$$
\begin{aligned}
H & \rightarrow\left\{\text { (oriented) geodesics in } \mathbb{H}^{+}\right\}, \\
\vec{u} & \mapsto\left\{\vec{w} \in \mathbb{H}^{+}:\langle\vec{w}, \vec{u}\rangle=0\right\},
\end{aligned}
$$

of affine and linear duals, respectively, establish bijections.

For various computations, we shall employ various coordinate systems on $\mathbb{M}$ which are next described. By "Cartesian coordinates", we shall mean the standard coordinates relative to the basis $(1,0,0),(0,1,0),(0,0,1)$ on $\mathbb{R}^{3}$. The second family of coordinatizations we shall need arises from a specification of three linearly independent light-like vectors, say $\vec{e}, \vec{f}, \vec{g} \in L^{+}$, so that $\langle\vec{e}, \vec{f}\rangle=\langle\vec{f}, \vec{g}\rangle=\langle\vec{g}, \vec{e}\rangle=-1$. Such a basis $\vec{e}, \vec{f}, \vec{g}$ is called a "light-cone basis" with its corresponding "light-cone coordinates" on $\mathbb{M}$, and any two positively oriented light-cone bases are related by an element of $S O^{+}(1,2)$. Moreover, given vectors $\vec{e}$ and $\vec{f}$ as above, there is a unique completion $\vec{g} \in L^{+}$to a positively oriented light-cone basis $\vec{e}, \vec{f}, \vec{g}$. Of course, if $\vec{p}=x \vec{e}+y \vec{f}+z \vec{g} \in \mathbb{M}$, then

$$
\langle\vec{p}, \vec{p}\rangle=-2\{x y+y z+z x\} .
$$

In particular, we shall need the "standard" light-cone basis given by

$$
\vec{e}=2^{-1 / 2}(0,1,1), \quad \vec{f}=2^{-1 / 2}(0,-1,1), \quad \text { and } \quad \vec{g}=2^{-1 / 2}(2,0,2),
$$

so

$$
(1,0,0)=2^{-1 / 2}\{\vec{g}-\vec{e}-\vec{f}\}, \quad(0,1,0)=2^{-1 / 2}\{\vec{e}-\vec{f}\},
$$

and

$$
(0,0,1)=2^{-1 / 2}\{\vec{e}+\vec{f}\} .
$$


We shall also require several coordinatizations of $\mathbb{H}^{+}$as follows. Radial projection from $(0,0,-1)$ establishes an isometry between $\mathbb{H}^{+}$(with the metric inherited from $\langle\cdot, \cdot\rangle$ ) and the unit disc $\mathbb{D}$ at height zero in $\mathbb{M}$ (with the Poincaré metric $d \zeta=$ $\frac{2|d \zeta|}{1-|\zeta|^{2}}$ for $\left.\zeta \in \mathbb{D}\right)$. Giving $\mathbb{D}$ the coordinates $(\xi, \eta)$ relative to the standard basis $(1,0),(0,1)$ on the plane at height zero in $\mathbb{M}$, we find the following correspondences with Cartesian coordinates on $\mathbb{H}^{+}$:

$$
\begin{gathered}
\mathbb{H}^{+} \rightarrow \mathbb{D}, \\
(\xi, \eta)=\left(\frac{x}{1+z}, \frac{y}{1+z}\right),
\end{gathered}
$$

and

$$
\begin{gathered}
\mathbb{D} \rightarrow \mathbb{H}^{+}, \\
x=\frac{2 \xi}{1-\xi^{2}-\eta^{2}}=\frac{2 r \cos \theta}{1-r^{2}}, \\
y=\frac{2 \eta}{1-\xi^{2}-\eta^{2}}=\frac{2 r \sin \theta}{1-r^{2}}, \\
z=\frac{1+\xi^{2}+\eta^{2}}{1-\xi^{2}-\eta^{2}}=\frac{1+r^{2}}{1-r^{2}},
\end{gathered}
$$

where $r, \theta$ are the standard polar coordinates on the plane at height zero in $\mathbb{M}$. Thus, the transformation from polar coordinates on $\mathbb{D}$ to standard light-cone coordinates on $\mathbb{H}^{+}$is given by

$$
\begin{gathered}
\mathbb{D} \rightarrow \mathbb{H}^{+}, \\
r \exp (i \theta) \mapsto 2^{-1 / 2}\left(1-r^{2}\right)^{-1}\left\{\left[1+r^{2}+2 r(\sin \theta-\cos \theta)\right] \vec{e}\right. \\
\left.+\left[1+r^{2}-2 r(\sin \theta+\cos \theta)\right] \vec{f}+[2 r \cos \theta] \vec{g}\right\} .
\end{gathered}
$$

Finally, fix some light-cone basis $\vec{e}, \vec{f}, \vec{g}$ for $\mathbb{M}$. If $\vec{p}=x \vec{e}+y \vec{f}+z \vec{g}$, then we define "lambda coordinates" (in analogy to the lambda lengths in $\S 3$ of [P1])

$$
\alpha=-\langle\vec{p}, \vec{e}\rangle=y+z, \quad \beta=-\langle\vec{p}, \vec{f}\rangle=x+z, \quad \text { and } \quad \gamma=-\langle\vec{p}, \vec{g}\rangle=x+y,
$$

so that

$$
x=\frac{1}{2}\{\beta+\gamma-\alpha\}, \quad y=\frac{1}{2}\{\alpha+\gamma-\beta\}, \quad \text { and } \quad z=\frac{1}{2}\{\alpha+\beta-\gamma\},
$$

and $-\langle\vec{p}, \vec{p}\rangle=\frac{1}{2}\left\{2[\alpha \beta+\beta \gamma+\gamma \alpha]-\left[\alpha^{2}+\beta^{2}+\gamma^{2}\right]\right\}$.

\section{REsults}

To begin, we have

Proposition 1. Suppose that $q \in \mathbb{H} \cup(L-\{\overrightarrow{0}\})$, and consider the function

$$
v(\vec{p} ; \vec{q})=\frac{\langle\vec{q}-\vec{p}, \vec{q}\rangle}{1+h(\vec{p})}, \quad \text { for } \vec{p} \in \mathbb{H}^{+} .
$$

Then $\log v$ is harmonic in $\vec{p}$. It follows that

$$
\Psi\left(\vec{p} ; \vec{q}_{1}, \vec{q}_{2}\right)=\log \frac{\left\langle\vec{q}_{1}-\vec{p}, \vec{q}_{1}\right\rangle}{\left\langle\vec{q}_{2}-\vec{p}, \vec{q}_{2}\right\rangle}
$$

is harmonic in $\vec{p}$ for any $\vec{q}_{1}, \vec{q}_{2} \in \mathbb{H} \cup(L-\{\overrightarrow{0}\})$. 
Proof. Given $\vec{p} \in \mathbb{H}^{+}, \vec{q} \in \mathbb{M}$, define $u(\vec{p}, \vec{q})=\langle\vec{q}-\vec{p}, \vec{q}\rangle$ and set

$$
\begin{gathered}
\vec{p}=x \vec{e}+y \vec{f}+z \vec{g}, \\
\vec{q}=a \vec{e}+b \vec{f}+c \vec{g},
\end{gathered}
$$

where $\vec{e}, \vec{f}, \vec{g}$ is the standard light-cone basis. We compute

$$
\begin{aligned}
& 2\{a b+b c+c a\}+u(\vec{p} ; \vec{q})=a(y+z)+b(x+z)+c(x+y) \\
& \quad=2^{-1 / 2}\left(1-r^{2}\right)^{-1}\left\{a\left[r^{2}+1-2 r \sin \theta\right]+b\left[r^{2}+2 r \sin \theta\right]+2 c\left[r^{2}+1-2 r \cos \theta\right]\right\} \\
& \quad=2^{-1 / 2} \frac{1+r^{2}}{1-r^{2}}\{a+b+2 c\}+2^{+1 / 2} \frac{r}{1-r^{2}}\{(b-a) \sin \theta-2 c \cos \theta\} .
\end{aligned}
$$

Of course,

$$
1+h(\vec{p})=1+\frac{1+r^{2}}{1-r^{2}}=\frac{2}{1-r^{2}},
$$

SO

$$
\begin{aligned}
v(\vec{p} ; \vec{q})= & \frac{u(\vec{p} ; \vec{q})}{1+h(\vec{p})} \\
= & r^{2}\left[2^{-3 / 2}(a+b+2 c)+a b+b c+c a\right] \\
& \quad+2^{-1 / 2} r[(b-a) \sin \theta-2 c \cos \theta]+\left[2^{-3 / 2}(a+b+2 c)-\{a b+b c+c a\}\right] \\
= & A r^{2}+B r+C,
\end{aligned}
$$

where $A$ and $C$ are independent of $\theta$, so

$$
v_{r r}=2 A, \quad v_{r}=2 A r+B, \quad v_{\theta \theta}=-B r,
$$

and

$$
v_{\theta}=2^{-1 / 2} r[2 c \sin \theta+(b-a) \cos \theta] .
$$

We find

$$
\begin{aligned}
v^{2} \Delta \log v= & {\left[A r^{2}+B r+C\right]\left[2 A+\frac{2 A r+B}{r}-\frac{B}{r}\right] } \\
& -[2 A r+B]^{2}-\frac{1}{2 r}[2 c \sin \theta+(b-a) \cos \theta] \\
= & 4 A\left[A r^{2}+B r+C\right]-\left[4 A^{2} r^{2}+B^{2}+4 A B r\right] \\
& -\left[2 c^{2} \sin ^{2} \theta+\frac{(b-a)^{2}}{2} \cos ^{2} \theta+2 c(b-a) \cos \theta \sin \theta\right] \\
= & 4 A C-\frac{1}{2}\left[(b-a)^{2}+4 c^{2}\right] \\
= & -4(a b+b c+c a)^{2}+2(a b+b c+c a) \\
= & -\langle\vec{q}, \vec{q}\rangle(1+\langle\vec{q}, \vec{q}\rangle) \\
= & 0 \quad \text { for } \vec{q} \in \mathbb{H} \cup(L-\{\overrightarrow{0}\}),
\end{aligned}
$$

as was asserted. 
Remark. Notice that for any $\vec{q} \in \mathbb{M}$ the function $v(\vec{p} ; \vec{q})$ satisfies the partial differential equation

$$
v^{2} \Delta \log v=\frac{v^{2}}{r^{2}}\left[r^{2} v_{r r}+r v_{r}+v_{\theta \theta}\right]=-\langle\vec{q}, \vec{q}\rangle(1+\langle\vec{q}, \vec{q}\rangle)
$$

of the "conformal factor" (cf. Poincaré's solution to the Yamabe problem in one complex dimension). In particular, if $\vec{q} \in \mathbb{H}^{+}$, then Green's function on $\mathbb{H}^{+}$with pole $\vec{q}$ is given by

$$
G(\vec{p}, \vec{q})=\frac{1}{2} \log \frac{\langle\vec{p}, \vec{q}\rangle-1}{\langle\vec{p}, \vec{q}\rangle+1}, \quad \text { for } p \in \mathbb{H}^{+} .
$$

Our next result is

Proposition 2. Suppose that $\vec{e}, \vec{f}, \vec{g}$ is a positively oriented light-cone basis on $\mathbb{M}$ with corresponding lambda coordinates $\alpha=-\langle\vec{p}, \vec{e}\rangle, \beta=-\langle\vec{p}, \vec{f}\rangle, \gamma=-\langle\vec{p}, \vec{g}\rangle$, for $\vec{p} \in \mathbb{H}^{+}$. Then the function

$$
\Phi(\vec{p})=\Phi_{\vec{e}, \vec{p}}(\vec{p})=\frac{1}{\alpha}\left\{\sqrt{-1}+2^{-1 / 2}[\alpha+\beta-\gamma]\right\}
$$

is an analytic function on $\mathbb{H}^{+}$. Furthermore, $\log |\Phi(\vec{p})|=\log \frac{2 \beta}{\alpha}$.

Proof. We will work in the upper half-space model $\mathcal{U}$ for the hyperbolic plane choosing a point in the circle $S_{\infty}^{1}$ at infinity (or, equivalently, a ray in $L^{+}$) which corresponds to the point at infinity in $\mathcal{U}$. We need the following two results.

Lemma 1. If $\vec{u} \in L^{+}$and $\vec{p} \in \mathbb{H}^{+}$, then $\langle\vec{u}, \vec{p}\rangle=-\exp (d)$, where $d$ is the signed hyperbolic distance from $\vec{p}$ to the horocycle $H(\vec{u})$ corresponding to $\vec{u} ; d$ is taken with the negative sign if $\vec{p}$ is inside $H(\vec{u})$ and with the positive sign if $\vec{p}$ is outside $H(\vec{u})$.

Lemma 2. Suppose that $G$ is an oriented geodesic in $\mathbb{H}^{+}$which corresponds to the point $\vec{q} \in H$, and let $d$ denote the signed hyperbolic distance from $G$ to a point $\vec{p} \in \mathbb{H}^{+}$; $d$ is taken with positive (negative) sign if $\vec{p}$ lies to the right (left, respectively) of the oriented geodesic $G$. In this case, we have

$$
\sinh (d)=\langle\vec{p}, \vec{q}\rangle \text {. }
$$

Furthermore, if $d^{\prime}$ denotes the signed hyperbolic distance from $(x, y) \in \mathcal{U}$ to the imaginary axis (where $d^{\prime}$ is given the sign of $x$ ), then $\sinh \left(d^{\prime}\right)=\frac{x}{y}$.

The proof of Lemma 1 is essentially identical to the proof of Lemma 2.1 of [P1] and is given in Lemma 2.2 of [P3]. Lemma 2 is standard, and the proof is left as an exercise.

Now, suppose that $\vec{u} \in L^{+}$, and consider the lambda coordinate $\alpha=-\langle\vec{p}, \vec{u}\rangle$, for $\vec{p} \in \mathbb{H}^{+}$. Map $\mathbb{H}^{+}$to $\mathcal{U}$ in such a way that the point in $S_{\infty}^{1}$ corresponding to $\vec{u}$ maps to infinity. The horocycle $H(\vec{u})$ thus corresponds to the locus $\left\{y=y_{0}\right\} \subset \mathcal{U}$ for some $y_{0}>0$. The distance from a point $(x, y) \in \mathcal{U}$ to this locus is $\left|\log \frac{y}{y_{0}}\right|$, and it follows from Lemma 1 that $\alpha=\exp \left( \pm\left|\log \frac{y}{y_{0}}\right|\right)$, taken with negative sign for $y>y_{0}$ and with positive sign for $y<y_{0}$. Thus, $\alpha^{-1}=\frac{y}{y_{0}}$ is a harmonic function on $\mathbb{H}^{+}$.

It follows from the second part of Lemma 2 that the harmonic conjugate of $\alpha^{-1}$ is

$$
\frac{x}{y_{0}}=\frac{x}{y} \frac{y}{y_{0}}=\alpha^{-1} \sinh (d)
$$


where $d$ is the signed distance to the imaginary axis. We next compute $\sinh (d)$ in lambda coordinates. To this end, complete $\vec{u}$ to a light-cone basis $\vec{u}, \vec{v}, \vec{w}$ so that $2^{-1 / 2}(\vec{u}+\vec{v}-\vec{w}) \in \mathbb{H}$ corresponds to the geodesic asymptotic to $\vec{u}$ and $\vec{v}$. It follows from the first part of Lemma 2 that

$$
\sinh (d)=-2^{-1 / 2}\langle\vec{u}+\vec{v}-\vec{w}, \vec{p}\rangle=2^{-1 / 2}(\alpha+\beta-\gamma),
$$

where $\beta=-\langle\vec{p}, \vec{v}\rangle$ and $\gamma=-\langle\vec{p}, \vec{w}\rangle$. Thus, the harmonic conjugate of $\alpha^{-1}$ is $2^{-1 / 2} \alpha^{-1}(\gamma-\alpha-\beta)$, proving the analyticity of the function $\Phi$ in Proposition 2 .

Finally, notice that

$$
\frac{(\alpha+\beta-\gamma)^{2}}{2}=2 \alpha \beta-1
$$

since $\vec{p} \in \mathbb{H}^{+}$, so $\Phi$ has norm squared $\frac{2 \beta}{\alpha}$, as was asserted.

Remark. In fact, the analytic function in Proposition 2 is just the function $z$ on $\mathcal{U}$ suitably pulled back to $\mathbb{H}^{+}$.

To close, we suppose that $g$ is a real-valued function on $L^{+}$which is constant on rays, so $g$ could be regarded as a function on the unit circle in the natural way. Our promised picture of Poisson kernel in Minkowski space follows.

Proposition 3. The function

$$
u(\vec{p})=\frac{1}{2 \pi} \int_{\vec{q} \in \gamma} \frac{-g(\vec{q})}{\langle\vec{q}, \vec{p}\rangle}|d \vec{q}|
$$

is a harmonic function of $\vec{p} \in \mathbb{H}^{+}$, where $\gamma$ is the contour in $L^{+}$which bounds the unit disk at height one in $\mathbb{M}$.

Proof. If $\vec{p}$ lies in $\mathbb{H}^{+}$with projection $w$ to $\mathbb{D}$, then one calculates as above that

$$
1-|w|^{2}=\frac{2}{1+h(\vec{p})}
$$

furthermore, if $\vec{q}=(\xi, \eta, 1) \in \gamma$ with corresponding $w^{\prime}=(\xi, \eta)$ in the boundary of $\mathbb{D}$, then one similarly calculates

$$
\left|w-w^{\prime}\right|=\frac{-2\langle\vec{q}, \vec{p}\rangle}{1+h(\vec{p})} .
$$

It follows that the Poisson kernel $\left(1-|w|^{2}\right) /\left|w-w^{\prime}\right|$ in $\mathbb{D}$ is indeed given by the kernel above, as was claimed.

\section{Closing Remarks}

We briefly discuss Poincaré series in the framework of this paper. As mentioned in the introduction, we associate to a (decorated) Riemann surface a certain convex body $C$ in $\mathbb{M}$. Indeed, all of the vertices of $C$ lie in $L^{+}$(at least generically), the faces of $C$ are triangles, and $C$ is a $\Gamma$-invariant convex body (regarding a Fuchsian group $\Gamma<S O^{+}(1,2)$ underlying the Riemann surface as acting on $\mathbb{M}$ ). In order to produce automorphic forms of various weights, one might associate an appropriate form to each face of $C$ (say using the elementary formulas of this note) summing over the faces in such a way that the limit exists.

In particular, for each topological type of punctured surface, there is a certain canonical finite family of (unmarked) conformal classes called "centers"; see $\S 6$ of [P1]. In fact, a Fuchsian group underlying a center is arithmetic (it leaves invariant 
the Farey tesselation), and there is an associated convex body $C_{\infty} \subset \mathbb{M}$ which corresponds to each and every center. There is, furthermore, a linear recursion for the vertices of $C_{\infty}$ in $L^{+}$, and one might thus attempt the more special problem of computing harmonic functions only for these special conformal types.

\section{REFERENCES}

[EP] D. B. A. Epstein and R. C. Penner, Euclidean decompositions of noncompact hyperbolic manifolds, Jour. Diff. Geom. 27 (1988), 67-80. MR 89a:57020

[P1] R. C. Penner, The decorated Teichmüller space of punctured surfaces, Comm. Math. Phys. 113 (1987), 299-339. MR 89h:32044

[P2] - Calculus on moduli space, Contemp. Math. 74 (1988), 277-293. MR 90a:32029

[P3] _ An arithmetic problem in surface geometry, The Moduli Space of Curves (Texel Island, 1994), Progr. Math., vol. 129, Birkhäuser, Boston, MA, 1995, pp. 427-466. CMP 96:04

Department of Mathematics, University of Southern California, Los Angeles, CalIFORNIA 90089

Current address, P. F. Glezen: ISSC, Inc., One Market Plaza, San Francisco, California 94105

E-mail address, R. C. Penner: rpenner@mathi.usc.edu 